\title{
Quantum ratchet effects induced by terahertz radiation in GaN-based two-dimensional structures
}

\author{
W. Weber, ${ }^{1}$ L. E. Golub, ${ }^{2}$ S. N. Danilov, ${ }^{1}$ J. Karch, ${ }^{1}$ C. Reitmaier, ${ }^{1}$ B. Wittmann, ${ }^{1}$ V. V. Bel'kov, ${ }^{2}$ E. L. Ivchenko, ${ }^{2}$ \\ Z. D. Kvon, ${ }^{3}$ N. Q. Vinh, ${ }^{4}$ A. F. G. van der Meer, ${ }^{4}$ B. Murdin,${ }^{5}$ and S. D. Ganichev ${ }^{1, *}$ \\ ${ }^{1}$ Terahertz Center, University of Regensburg, 93040 Regensburg, Germany \\ ${ }^{2}$ A.F. Ioffe Physico-Technical Institute, Russian Academy of Sciences, 194021 St. Petersburg, Russia \\ ${ }^{3}$ Institute of Semiconductor Physics, Russian Academy of Sciences, 630090 Novosibirsk, Russia \\ ${ }^{4}$ FOM Institute for Plasma Physics "Rijnhuizen," P.O. Box 1207, NL-3430 BE Nieuwegein, The Netherlands \\ ${ }^{5}$ University of Surrey, Guildford GU2 7XH, United Kingdom
}

(Received 30 March 2008; revised manuscript received 6 May 2008; published 4 June 2008)

\begin{abstract}
Photogalvanic effects are observed and investigated in wurtzite (0001)-oriented GaN/AlGaN lowdimensional structures excited by terahertz radiation. The structures are shown to represent linear quantum ratchets. Experimental and theoretical analysis exhibits that the observed photocurrents are related to the lack of an inversion center in the GaN-based heterojunctions.
\end{abstract}

DOI: 10.1103/PhysRevB.77.245304

PACS number(s): 73.21.Fg, 78.67.De, 73.63.Hs

\section{INTRODUCTION}

In recent years, physics of quantum ratchets draw a growing attention. In mechanics, a ratchet is a device that is used to restrict motion in one direction while permitting it in another. In general, one means by a ratchet any sort of asymmetric potential or a potential lacking a center of the spatial inversion. At the turn of 1980s and 1990s, it was understood that unbiased nonequilibrium noncentrosymmetric systems can generate transport of particles, and this conception has roots in different fields of physics, chemistry, and biology. In mechanical, electronic, optical, and biological systems, a particle, classical, or quantum, charged or neutral, propagating in a periodic potential with broken centrosymmetry and subjected to an ac force exhibits a net dc macroscopic flow. Examples are electrons in solids, Abrikosov vortices in superconductors, and biological motor proteins. ${ }^{1}$ One of implementations of the ratchet phenomenon is a linear photogalvanic effect (LPGE). It represents a generation of a dc electric current under absorption of linearly polarized light in unbiased crystals of piezoelectric classes. Glass et al. ${ }^{2}$ were the first to attribute a photoinduced current observed in ferroelectric $\mathrm{LiNbO}_{3}$ to a novel photogalvanic effect and proposed the first correct model for its microscopic qualitative interpretation. A consistent quantitative theory of LPGE was developed by Belinicher and Sturman followed by other theorists (see Refs. 3 and 4).

Noncentrosymmetric bulk semiconductors and heterostructures are natural quantum ratchets and the further studies of LPGE in these systems allow one to elucidate the whole problem of the ratchet effect. In particular, a current of the LPGE consists of two contributions, ballistic and shift, which as a rule are comparable in the order of magnitude. ${ }^{5}$ It is important to note that similar two contributions to the electric current, called the skew-scattering and side-jump currents, do exist in the anomalous Hall effect (see the recent review by $\operatorname{Sinitsyn}^{6}$ ). Another important point is that one should include into the light absorption process contributing to the ballistic current an additional simultaneous scattering from a static defect or a phonon compared to the light- induced optical transition, leading to the shift current. In GaN-based structures first observation of LPGE has been reported quite recently. ${ }^{7-12}$ Here, we present results of detailed experimental and theoretical investigation of LPGE in heterostructures based on GaN and its alloys with AlN. The commercial fabrication of blue and green light-emitting diodes have led to well established technological procedures of epitaxial preparation of these heterostructures and initiated a great activity on investigations of their properties. ${ }^{13}$ The photogalvanics serves as a solid bridge between transport and optics and, therefore, reveals both transport and optical properties of the systems under study.

\section{SAMPLES AND EXPERIMENTAL METHODS}

The experiments are carried out on (0001)-oriented wurtzite $n-\mathrm{GaN} / \mathrm{Al}_{0.3} \mathrm{Ga}_{0.7} \mathrm{~N}$ heterojunctions grown by metalorganic chemical vapor deposition on $C$-plane sapphire substrates (for details of growth, see Ref. 7). The thickness of the AlGaN layers was varied between 30 and $100 \mathrm{~nm}$. An undoped $33 \mathrm{~nm}$ thick GaN buffer layer grown under a pressure of $40 \mathrm{~Pa}$ at a temperature of $550{ }^{\circ} \mathrm{C}$ is followed by an undoped $\mathrm{GaN}$ layer $(\sim 2.5 \mu \mathrm{m})$ grown under $40 \mathrm{~Pa}$ at $1025^{\circ} \mathrm{C}$; the undoped $\mathrm{Al}_{0.3} \mathrm{Ga}_{0.7} \mathrm{~N}$ barrier was grown under $6.7 \mathrm{~Pa}$ at $1035^{\circ} \mathrm{C}$. The mobility and density in the twodimensional (2D) electron gas measured at room temperature are $\mu \approx 1200 \mathrm{~cm}^{2} / \mathrm{Vs}$ and $N_{s} \approx 10^{13} \mathrm{~cm}^{-2}$, respectively. To measure the photocurrent two pairs of contacts are centered at opposite sample edges with the connecting lines along the axes $x \|[1 \overline{1} 00]$ and $y \|[11 \overline{2} 0]$ (see inset in Fig. 1). The current generated by the light in the unbiased samples is measured via the voltage drop across a $50 \Omega$ load resistor in a closedcircuit configuration. The voltage is recorded with a storage oscilloscope.

The photocurrents were induced by indirect intrasubband (Drude-type) optical transitions in the lowest size-quantized subband. The emission from a terahertz molecular laser optically pumped by a transversely excited atmospheric pressure (TEA) $\mathrm{CO}_{2}$ laser $^{14}$ is used for the optical excitation. 


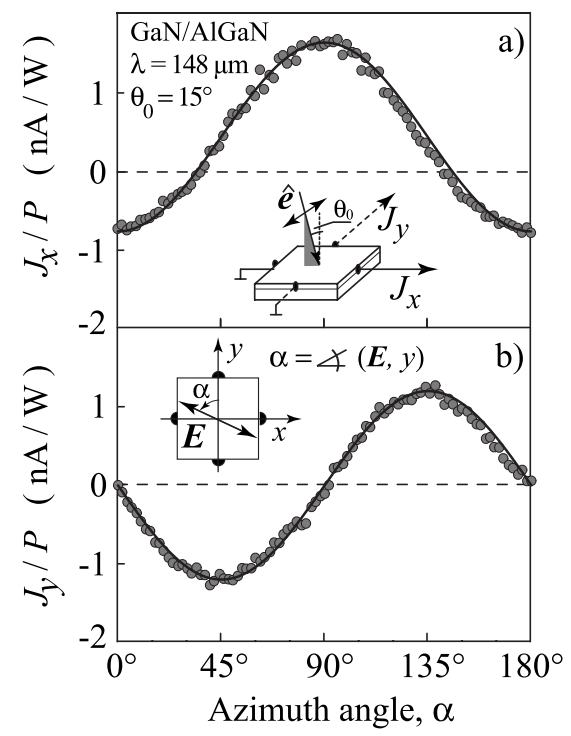

FIG. 1. Photocurrent as a function of angle $\alpha$ measured at room temperature at oblique incidence $\left(\theta_{0}=15^{\circ}\right)$ in the (a) longitudinal $\left(J_{x}\right)$ and (b) transverse $\left(J_{y}\right)$ geometries. Photocurrent is excited by linearly polarized radiation with wavelength $\lambda=148 \mu \mathrm{m}$ and power $P \approx 5 \mathrm{~kW}$. The full lines are fits to Eqs. (6). To get agreement with experiments, we used one fitting parameter $J_{0} \propto \chi$ and introduced an offset $J_{\text {offset }}$ for the current $J_{x}$ detected in the direction of light propagation. The inset shows the experimental geometry. An additional inset in the lower panel displays the sample and the radiation electric field viewing from the source of radiation side.

With $\mathrm{NH}_{3}, \mathrm{D}_{2} \mathrm{O}$, and $\mathrm{CH}_{3} \mathrm{~F}$ as an active media, we could obtain radiation pulses of duration of $\simeq 100$ ns with wavelengths $\lambda=77,90.5,148,280,385$, and $496 \mu \mathrm{m}$ and a power $P \simeq 5 \mathrm{~kW}$. Radiation in both the normal- and obliqueincidence geometries was applied with the angle of incidence $\theta_{0}$ varying from $-30^{\circ}$ to $+30^{\circ}, \theta_{0}=0$, corresponding to the normal incidence and laser beam lying in the $(x z)$ plane (see insets in Figs. 1, 2, and 4).

Optically pumped molecular lasers emit linearly polarized radiation with the polarization plane determined by that of the pump light: the electric field vector of the terahertz radiation $\boldsymbol{E}_{l}$ generated by the molecular laser can be either parallel (like for the lines $\lambda=90.5,148$, and $280 \mu \mathrm{m}$ ) or orthogonal (lines 77, 385, and $496 \mu \mathrm{m}$ ) to the polarization vector of the pump beam depending on the angular momentum selection rules for the pump and the terahertz transitions in the active media. ${ }^{14}$

In the experiments, the plane of polarization of the radiation incident on the sample was rotated applying $\lambda / 2$ plates, which enabled us to vary the azimuth angle $\alpha$ from $0^{\circ}$ to $180^{\circ}$ covering all possible orientations of the electric field component in the $(x y)$ plane. Hereafter, the angle $\alpha=0$ is chosen in such a way that the incident light polarization is directed along the $y \|[11 \overline{2} 0]$ direction [see inset in Fig. 1(b)].

To investigate the photogalvanic effects, we also use elliptically polarized light. In this case, the polarization of the laser beam is modified from linear to elliptical by means of crystal quartz $\lambda / 4$ plates. The counterclockwise rotation (viewing from the laser side) of the optical axis of the quarter-wave plate by the angle $\varphi_{p}$ results in the variation of

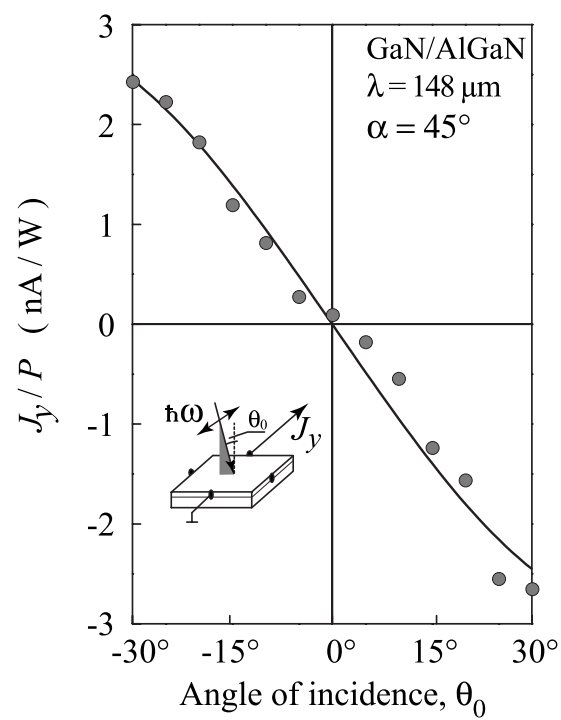

FIG. 2. Photocurrent as a function of angle of incidence $\theta_{0}$ measured at room temperature in the transverse geometry and azimuth angle $\alpha=45^{\circ}$. Photocurrent is excited by radiation with wavelength $\lambda=148 \mu \mathrm{m}$ and power $P \approx 5 \mathrm{~kW}$. The full line is the fit to Eqs. (6) with the same fitting parameter $J_{0}$ as used in Fig. 1. The inset shows the experimental geometry.

the radiation helicity as $P_{\text {circ }}=-\sin 2 \varphi_{p}$. Here, the angle $\varphi_{p}=0$ is chosen for the position of the quarter-wave plate optical axis coinciding with the incoming laser polarization, in which case, the linear polarization degree is given by fourth harmonics of $\varphi_{p}$.

A series of measurements is carried out by making use of the frequency tunability and short pulse duration of the free electron laser "FELIX" at FOM-Rijnhuizen in the Netherlands operated in the spectral range between 70 and $120 \mu \mathrm{m} .{ }^{15}$ The output pulses of light from the FELIX were chosen to be $\approx 6$ ps long, separated by $40 \mathrm{~ns}$ in a train (or "macropulse") of $7 \mu$ s duration. The macropulses had a repetition rate of $5 \mathrm{~Hz}$.

\section{EXPERIMENTAL RESULTS}

Irradiating the (0001)-grown GaN/AlGaN heterojunction by polarized light at oblique incidence, as sketched in the inset to Fig. 1(a), causes a photocurrent signal measured across a contact pair. The width of the photocurrent pulses is about $100 \mathrm{~ns}$, which corresponds to the terahertz laser pulse duration. The signal depends on the light polarization, and all characteristic polarization properties persist from 4.2 to 300 $\mathrm{K}$. The experimental data presented below are obtained at room temperature. The effect is observed for all the wavelengths applied (between 77 and $496 \mu \mathrm{m}$ ). We use two geometries: the longitudinal geometry $\left(J_{x}\right.$ in the Fig. 1), in which the photocurrent is measured in the direction along in-plane component $\hat{\boldsymbol{e}}_{\|}$and the transverse geometry $\left(J_{y}\right.$ in the Fig. 1), where the signal is detected in the direction normal to the light propagation unit vector $\hat{\boldsymbol{e}}$ [see the inset in Fig. $1(\mathrm{a})]$.

Figure 1 shows the dependence of the photocurrent on the azimuth angle $\alpha$ for both experimental geometries obtained 


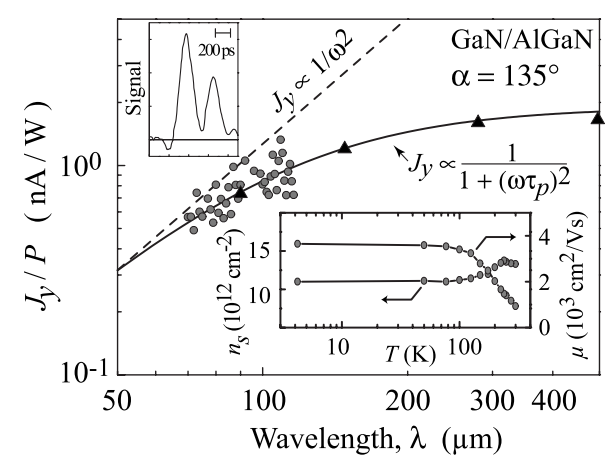

FIG. 3. Spectral dependence of the transverse photocurrent $J_{y}$ measured at room temperature at oblique incidence $\left(\theta_{0}=15^{\circ}\right)$ for azimuth angle $\alpha=135^{\circ}$. The data are obtained with the free electron laser FELIX (dots) and with molecular optically pumped laser (triangles). The full line shows fit to Eq. (1). The fit is obtained using $\tau_{p}$ as an adjustable parameter and a scaling the whole dependence by the ordinate. The dashed line shows $J_{y} \propto \omega^{-2}$ for comparison. The inset in the left upper corner shows the temporal structure of the current in response to the radiation of FELIX. The inset in the right corner shows the temperature dependences of the carrier density and the mobility.

at the positive incidence angle $\theta_{0}=15^{\circ}$ and $\hat{\boldsymbol{e}}_{\|} \| x$. The polarization dependence of the current in the longitudinal geometry is well fitted by $J_{x}=J_{0}(1-\cos 2 \alpha)+J_{\text {offset }}$, while for the transverse geometry, we have $J_{y}=-J_{0} \sin 2 \alpha$. Note that the offset contribution $J_{\text {offset }}$ is observed in the longitudinal geometry only. Below, in Sec. IV B, we will demonstrate that exactly these dependences follow from the theory. As a function of the incidence angle, the photocurrent changes sign at $\theta_{0} \approx 0$. This is demonstrated in Fig. 2, which shows the dependence $J_{y}\left(\theta_{0}\right)$ for the fixed azimuth angle $\alpha=45^{\circ}$. A similar dependence is also detected for the longitudinal current $J_{x}$. We note that, like the polarization dependent contribution to the photocurrent, the detected offset current in the longitudinal geometry $J_{\text {offset }}$ inverts its direction when the incidence angle changes its sign.

The wavelength dependence of the photocurrent obtained in transverse geometry at azimuth angle $\alpha=135^{\circ}$ and angle of incidence $\theta_{0}=15^{\circ}$ is shown in Fig. 3. The data are measured both on FELIX (dots) and on molecular laser (triangles). Fitting the data to the well known spectral behavior of the Drude absorption, ${ }^{16,17}$

$$
J_{y}(\omega) \propto \eta(\omega) \propto \frac{1}{1+\left(\omega \tau_{p}\right)^{2}},
$$

we obtain that the spectral behavior of the photocurrent can reasonably be described by this equation (see full line in Fig. $3)$. For the fit we used $\tau_{p}$ as an adjustable parameter and scaled the whole dependence by the ordinate. Analyzing this dependence, we obtained that the momentum relaxation time controlling absorption is about $0.05 \mathrm{ps}$. This value is twice shorter than the transport time $(\simeq 0.1 \mathrm{ps})$ extracted from the mobility measurements at room temperature. We attribute the shortening of the momentum relaxation time by electron gas heating due to absorption of the intense terahertz radiation. The reduction in the mobility due to heating stems from

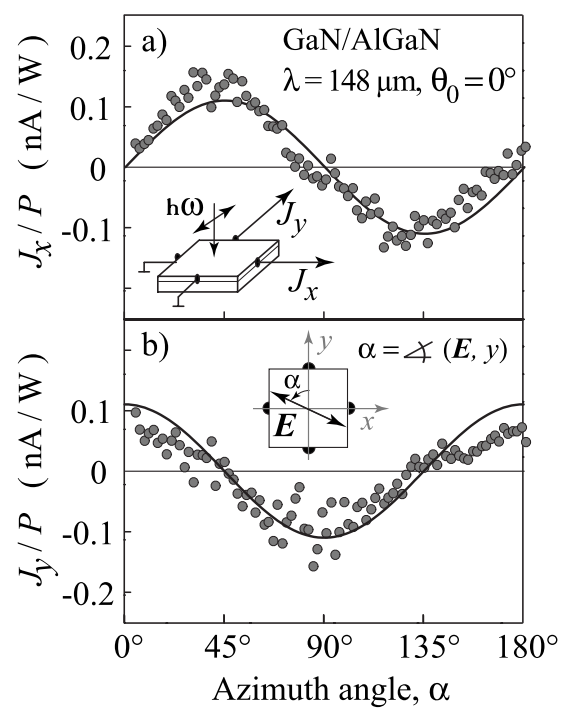

FIG. 4. Photocurrent as a function of angle $\alpha$ measured at normal incidence $\left(\theta_{0}=0\right)$ in (a) $x$ and (b) $y$ crystallographic directions. Photocurrent is excited by linearly polarized radiation with wavelength $\lambda=148 \mu \mathrm{m}$ and power $P \approx 5 \mathrm{~kW}$. The full lines are fits to Eq. (5). To get agreement with experiments, we used one fitting parameter $J_{1} \propto \chi^{\prime}$. The inset shows the experimental geometry. An additional inset in the lower panel displays the sample and the radiation electric field viewing from the source of radiation side.

enhancement of scattering by phonons under increase of temperature. Using short 6 ps pulses of FELIX, we observed that the response time is determined by the time resolution of our setup but it is at most 100 ps or shorter (see the inset in the left upper corner of Fig. 3). This fast response is typical for photogalvanics where the signal decay time is expected to be of the order of the momentum relaxation time ${ }^{3,4,14}$ being in our samples at room temperature of the order of 0.1 ps.

In addition to the signal excited by the radiation at oblique incidence, we also detected a small photocurrent at normal incidence. The polarization behavior of this signal is shown in Fig. 4. In this setup, photocurrent dependences can be well fitted by $J_{x}=J_{1} \sin 2 \alpha$ and $J_{y}=J_{1} \cos 2 \alpha$ (see also Sec. IV A). Rotating the sample around $z$ axis, we proved that the current direction is solely determined by the orientation of radiation electric field relative to the in-plane crystallographic axes. We note that this contribution is about 1 order of magnitude smaller than that at oblique incidence even at small angles $\theta_{0} \approx 15^{\circ}$.

Besides investigations of photocurrent in response to linearly polarized radiation, we also performed measurements under excitation with elliptically polarized light. Such experiments are of particular interest because such radiation, in partially circularly polarized light, has previously been used for investigation of the circular photogalvanic effect (CPGE) ${ }^{4,7,14}$ which coexist with the LPGE yielding beatings in $\varphi_{p}$ dependence. The CPGE is characterized by a photocurrent whose direction is changed upon reversal of the radiation helicity. It has been observed in GaN-based structures demonstrating a substantial structural inversion asymmetry (SIA) in this wide band gap material. ${ }^{7,9-11}$ We have shown 

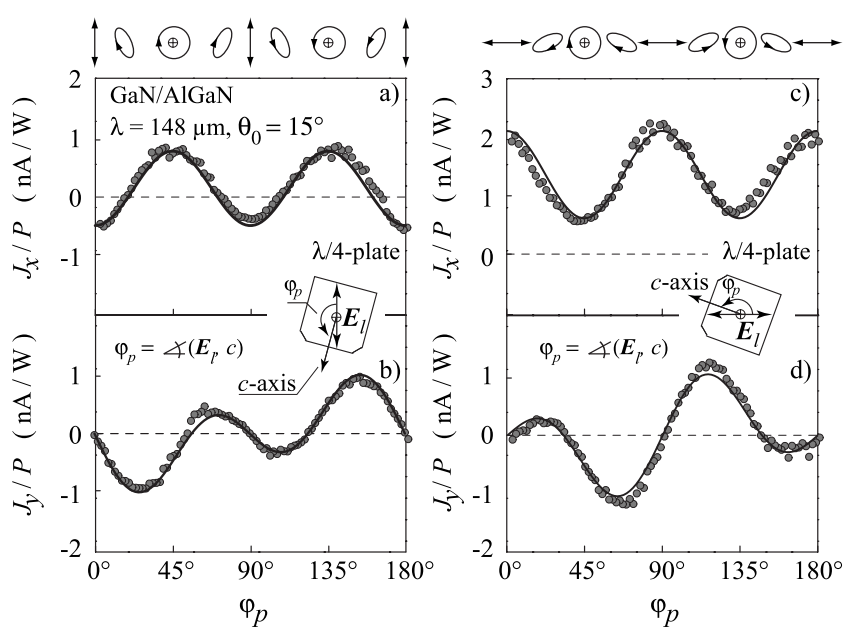

FIG. 5. Photocurrent as a function of angle $\varphi_{p}$ measured at oblique incidence $\left(\theta_{0}=15^{\circ}\right)$ in the longitudinal $\left[J_{x}\right.$ in (a) and (c)] and transverse $\left[J_{y}\right.$ in (b) and (d) $]$ geometries at wavelength $\lambda$ $=148 \mu \mathrm{m}$ and power $P \approx 5 \mathrm{~kW}$. The ellipticity of the radiation is varied by passing linearly polarized laser radiation $\left(\boldsymbol{E}_{l}\right)$ through a quarter-wave plate (see insets in the left and right panels). The left panels show transverse and longitudinal photocurrents measured in experimental setup with $\boldsymbol{E}_{l}$ perpendicular to the incidence plane ( $s$ polarization at $\varphi_{p}=0$, see inset in the left panel). Photocurrents obtained for $\boldsymbol{E}_{l}$ parallel to the incidence plane ( $p$ polarization at $\varphi_{p}=0$, see inset in the right panel) are shown in the right panel. The full lines are fits of the photocurrent to Eqs. (9) and (10) obtained correspondingly in the experimental setup sketched in the inset in the left and right panels. The fits are obtained using the same values of $J_{0}$ and $J_{\text {offset }}$ as in the experiments with linearly polarized radiation. The inset in the left panels shows the experimental geometry. On top the polarization, ellipses corresponding to various phase angles $\varphi_{p}$ are plotted viewing from the source of radiation.

that SIA in GaN-based heterostructures results in a spin splitting of subbands in $\boldsymbol{k}$ space, which was later confirmed by magnetotransport measurements. ${ }^{18-21}$ The Rashba spin splitting due to SIA, which is not expected in wide band semiconductors, in GaN/AlGaN heterostructures is caused by a large piezoelectric effect, ${ }^{22}$ which yields a strong electric field at the GaN/AlGaN interface and a strong polarization induced doping effect. ${ }^{23}$

Figures 5(a) and 5(b) demonstrate the dependences of the photocurrent on the angle $\varphi_{p}$ for positive angle $\theta_{0}$. In Fig. 5(a) transverse and longitudinal photocurrents are measured for the experimental setup with $\boldsymbol{E}_{l}$ perpendicular to the incidence plane ( $s$ polarization at $\left.\varphi_{p}=0\right)$. We find that the polarization dependences in this experiment are well fitted by $J_{x}$ $=J_{0}\left(1-\cos 4 \varphi_{p}\right) / 2+J_{\text {offset }}$ and for transverse geometry by $J_{y}=-J_{0} \sin 4 \varphi_{p} / 2+J_{2} \sin 2 \varphi_{p}$ (see Sec. IV B 2 for theoretical justification). We note that we used for fitting the same values of $J_{0}$ and $J_{\text {offset }}$ as in experiments with linearly polarized radiation.

As we discussed above, our laser can generate linearly polarized radiation either oriented along or perpendicularly to the polarization of the pump radiation. By that we change the position of the electric field of transformed beam relative to the optical axis of the quarter-wave plate, consequently changing the orientation of the ellipse as well as radiation

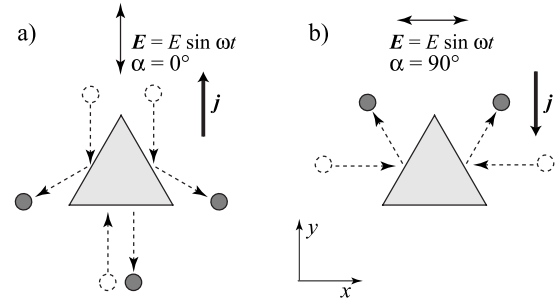

FIG. 6. Model of current generation due to asymmetry of specular elastic scattering by wedges. The field $\boldsymbol{E}$ results in directed motion of carriers shown by the dashed arrows. Due to asymmetric scattering a directed carrier flow and, therefore, electric current $j$ are generated. (a) and (b) sketch two relative orientations of wedges and the electric field $\boldsymbol{E}$ together with resulting dc current $\boldsymbol{j}$.

helicity of the beam at the sample. In order to check how this transformation influence results, we provided an additional experiment using laser beam with again $\lambda=148 \mu \mathrm{m}$ and $\hat{\boldsymbol{e}}_{\|} \| x$ but for $\boldsymbol{E}_{l}$ parallel to the incidence plane ( $p$ polarization at $\left.\varphi_{p}=0\right)$. Figures 5(c) and 5(d) show the data obtained in this geometry. In contrast to the results presented in Figs. 5(a) and 5(b), the photocurrent now shows another type of polarization dependences: $J_{x}=J_{0}\left(3+\cos 4 \varphi_{p}\right) / 2+J_{\text {offset }}$ and for transverse geometry $J_{y}=J_{0} \sin 4 \varphi_{p} / 2+J_{2} \sin 2 \varphi_{p}$. The fact that contribution given by $J_{2}$ did not change is not surprising because this term is due to radiation helicity which does not change: $P_{\text {circ }}=-\sin 2 \varphi_{p}$. The changes in contribution induced by the degree of linear polarization are caused by the fact that the orientation of corresponding ellipses at the sample at a given $\varphi_{p}$ is different.

The photocurrent polarization properties revealed experimentally both under normal and oblique incidences are explained in Sec. IV, where we propose a simple illustrative model to interpret the nature of the photocurrent under study and derive general phenomenological equations. The measured wavelength and temperature dependences of the LPGE current are described in Sec. V where a microscopic theory is developed.

\section{MODELS, PHENOMENOLOGY, AND POLARIZATION DEPENDENCES OF THE PHOTOCURRENTS}

The appearance of the LPGE can be visualized by a simple one-dimensional model of randomly distributed but identically oriented wedges acting as asymmetric scattering centers. $^{3,4,14}$ This model showing the generation of a photogalvanic current is relevant for $2 \mathrm{D}$ GaN/AlGaN structures excited by the radiation at normal incidence. In Fig. 6 a wedge with a base oriented along $x$ direction is depicted which obviously does not possess a center of inversion. In equilibrium the velocities of electrons are isotropically distributed. Application of an external in-plane alternating field $\boldsymbol{E}(t)=\boldsymbol{E}$ sin $\omega t$ adds an oscillatory motion along the electric field to the random thermal motion of the electrons. If the field points along the height of the wedges $(\boldsymbol{E} \| y$ or $\alpha=0)$, then the scattering results in a carrier flow in the $(-y)$ direction, yielding an electric current $j_{y}>0$ shown by the up arrow in Fig. 6(a). By that electron fluxes along the $x$ direction compensate each other and $j_{x}$ is absent. A variation of the 
relative direction between the electric field and the orientation of the wedges changes the direction of the carrier flow resulting in a characteristic polarization dependence. It may, e.g., reverse its direction, as it is shown in Fig. 6(b) for the field oriented along the base of the wedges, or rotate by $90^{\circ}$ in the case of $\boldsymbol{E}$ directed at $45^{\circ}$ to $y$ axis.

In order to describe the observed polarization and angle of incidence dependences, we derive here phenomenological equations for the photocurrents in 2D GaN-based structures and give a model for LPGE. The linear photogalvanic current density $\boldsymbol{j}$ is phenomenologically described by the following expression: $:^{3,4,14}$

$$
j_{\lambda}^{\mathrm{LPGE}}=\sum_{\mu \nu} \chi_{\lambda \mu \nu}\left(E_{\mu} E_{\nu}^{*}+E_{\nu} E_{\mu}^{*}\right) .
$$

Here, $\boldsymbol{E}$ is the electric field amplitude of the light wave and $\boldsymbol{\chi}$ is the third-rank tensor symmetric in the last two indices. The index $\lambda$ enumerates two in-plane coordinates $x$ and $y$, while $\mu$ and $\nu$ run over all three Cartesian coordinates.

The LPGE is allowed only in piezoelectric crystal classes of noncentrosymmetric media where nonzero components of the tensor $\boldsymbol{\chi}$ do exist. The point symmetry of wurtzite $2 \mathrm{D}$ electron systems confined in the [0001] direction is $\mathrm{C}_{3 v}$, which differs from the $\mathrm{C}_{6 v}$ symmetry of the bulk GaN due to the absence of translation along the growth axis $z$, i.e., because of the presence of interfaces. The coordinate frame $x, y, z$ chosen in the experimental setup means that the plane $(y z) \|(1 \overline{1} 00)$ coincides with the mirror-reflection planes $\sigma_{v}$ contained in the $\mathrm{C}_{3 v}$ point group. In this point group, the tensor $\boldsymbol{\chi}$ has two linearly independent components for $\lambda \neq z$,

$$
\chi \equiv \chi_{x x z}=\chi_{y y z}, \quad \chi^{\prime} \equiv \chi_{x x y}=\chi_{y x x}=-\chi_{y y y} .
$$

This means that the phenomenological equation (2) reduces to

$$
\begin{gathered}
j_{x}^{\mathrm{LPGE}}=\chi\left\{E_{x} E_{z}^{*}\right\}+\chi^{\prime}\left\{E_{x} E_{y}^{*}\right\}, \\
j_{y}^{\mathrm{LPGE}}=\chi\left\{E_{y} E_{z}^{*}\right\}+\chi^{\prime}\left(\left|E_{x}\right|^{2}-\left|E_{y}\right|^{2}\right),
\end{gathered}
$$

where $\left\{E_{\mu} E_{\nu}^{*}\right\}=E_{\mu} E_{\nu}^{*}+E_{\nu} E_{\mu}^{*}$.

\section{A. Normal incidence}

In our experiments, a linear photogalvanic current is observed at normal incidence (see Fig. 4). It follows from Eqs. (4) that while at normal incidence the photocurrent proportional to the constant $\chi$ vanishes because $E_{z}=0$, the contribution determined by the constant $\chi^{\prime}$ is nonzero. The observation of this photocurrent is an important result demonstrating substantial difference in symmetry between wurtzite 2D GaN(0001)-based structures with $C_{3 v}$ symmetry and zinc-blende GaAs- and InAs-based heterostructures having $C_{2 v}$ or $D_{2 d}$ symmetry, where the LPGE is forbidden at normal light incidence.

The polarization dependences for normal incidence follow from Eqs. (4) and are given by

$$
j_{x}=\chi^{\prime}\left(t_{0} E_{0}\right)^{2} \sin 2 \alpha,
$$

$$
j_{y}=\chi^{\prime}\left(t_{0} E_{0}\right)^{2} \cos 2 \alpha,
$$

where $t_{0}=2 /\left(n_{\omega}+1\right)$ is the amplitude transmission coefficient for normal incidence and $n_{\omega}$ is the refractive index of the medium (for $\mathrm{GaN}, n_{\omega}=2.3$ ). Both polarization dependences well describe our experimental data with one fitting parameter $\chi^{\prime}$ (see the solid lines in Fig. 4). This agreement clearly demonstrates the generation of LPGE current at normal incidence. We emphasize that the current direction depends on the orientation of the polarization plane with respect to the crystallographic axes $x$ and $y$. In the case that contacts are arbitrary oriented relative to the crystallographic directions, Eq. (5) holds but the phase shift appears. This is considered in Appendix A.

\section{B. Oblique incidence}

\section{Linear polarization}

Linear photogalvanic effect is also observed at oblique incidence. Moreover, comparison of Figs. 1 and 4 shows that even at a small incidence angle $\theta_{0}$, the photocurrent is substantially (by the order of magnitude) larger than that at normal incidence. While at normal incidence the photogalvanic current is solely described by the constant $\chi^{\prime}$ at oblique incidence, another term in Eq. (4) shows up. This term is determined by the second linearly independent constant $\chi$. The polarization dependence of this contribution in the geometry relevant to the experiment depicted in Fig. 1, where the incidence plane is chosen to be the plane $(x z)$ and the angle $\alpha$ is counted from $y$, is given by

$$
\begin{gathered}
j_{x}(\alpha)=\chi E_{0}^{2} t_{p}^{2} \cos \theta \sin \theta(1-\cos 2 \alpha), \\
j_{y}(\alpha)=-\chi E_{0}^{2} t_{p} t_{s} \sin \theta \sin 2 \alpha .
\end{gathered}
$$

Here, $\theta$ is the refraction angle related to the incidence angle $\theta_{0}$ by $\sin \theta=\sin \theta_{0} / n_{\omega}$, and $t_{s}$ and $t_{p}$ are the Fresnel amplitude transmission coefficients from vacuum to the structure for the $s$ - and $p$-polarized lights, respectively. ${ }^{24}$ These functions are shown in Fig. 1 by the solid lines. To get agreement with experiments, we used the above equations with one fitting parameter $\chi$ and introduced an offset for the current $J_{x}$ detected in the direction of the light propagation. Registration of these characteristic polarization dependences proves observation of LPGE at oblique incidence.

Comparing the results presented in Figs. 1 and 4, we can estimate of the ratio of different components of the tensor $\boldsymbol{\chi}$ for the studied structures. At $\theta \approx 0.1 \mathrm{rad}, t_{s} \approx t_{p} \approx t_{0}$, therefore from the ratio of the amplitudes in Figs. 1 and 4 equal to $\left|\chi^{\prime} / \chi \theta\right|$, we get $\left|\chi^{\prime} / \chi\right| \approx 10^{-2}$. This hierarchy of components of the third rank tensor $\chi_{\lambda \mu \nu}$ in the systems of $C_{3 v}$ symmetry is expectable; ${ }^{3}$ however, we demonstrate that the $\chi^{\prime}$-related effect is observable. While the effect described by the constant $\chi$ also exists in the bulk GaN as well as in the $C_{\infty v}$ group, the photocurrent proportional to $\chi^{\prime}$ arises only due to the reduced symmetry $C_{3 v}$ of the system, i.e., due to size quantization. Therefore, the latter contribution should increase at narrowing of the $2 \mathrm{D}$ layer.

Equations (6) show that the photogalvanic current should follow the dependence $t_{s} t_{p} \sin \theta$, in particular, reverse its di- 
rection upon inversion the angle of incidence. This behavior is indeed observed. Figure 2 shows the LPGE current dependence on the incidence angle $\theta_{0}$ obtained for $J_{y}$ at a fixed linear polarization of radiation $\left(\alpha=45^{\circ}\right)$. We note that the offset photocurrent observed in the longitudinal direction only is always directed antiparallel to $\hat{\boldsymbol{e}}_{\|}$. We ascribe the offset to the photon drag current which is linearly coupled to the photon momentum. This effect is out of scope of the present paper.

In our experiments, we also probe LPGE for the light directed along other crystallographic directions. Investigating transverse and longitudinal currents being perpendicular and parallel to the incidence plane, respectively, we observed that all polarization dependences are described by the same constant $\chi$ independently of the in-plane direction of the light propagation. The reason of this fact is that the current contribution proportional to $\chi$ is due to cone asymmetry being axially isotropic in the plane of our structures. Thus, this photocurrent is totally determined by the light polarization state and propagation direction, and it is independent of the incidence plane orientation relative to the in-plane crystallographic axes. For linearly polarized light, the photocurrent can be presented in the invariant form as $\boldsymbol{j}=2 \chi \boldsymbol{E}_{\|} E_{z}$, where $\boldsymbol{E}_{\|}$is the projection of the light polarization vector $\boldsymbol{E}$ onto the structure plane. It is seen that the LPGE current is always directed along $\boldsymbol{E}_{\|}$. By introducing the azimuth angle $\beta$ so that $\beta=0$ corresponds to the polarization perpendicular to the incidence plane ( $s$ polarization), we obtain for transverse $\left(\boldsymbol{j}_{\perp}\right)$ and longitudinal $\left(\boldsymbol{j}_{\|}\right)$currents

$$
\begin{gathered}
\boldsymbol{j}_{\perp}=\chi E_{0}^{2} t_{p} t_{s} \sin 2 \beta\left(\hat{\boldsymbol{e}_{\|}} \times \hat{\boldsymbol{z}}\right), \\
\boldsymbol{j}_{\|}=\chi E_{0}^{2} t_{p}^{2} \cos \theta(1-\cos 2 \beta) \hat{\boldsymbol{e}}_{\|},
\end{gathered}
$$

where $\hat{z}$ is the unit vector along the normal.

\section{Elliptical polarization}

Illumination of the structure by elliptically polarized radiation also results in generation of the LPGE current, which is determined by the linear polarization degree of the light. However, elliptically polarized radiation is also characterized by the nonzero helicity. This results in the additional effect solely determined by the degree of circular polarization, the circular photogalvanic effect. ${ }^{3,4,14}$ This effect is phenomenologically described by

$$
j_{\lambda}^{\mathrm{CPGE}}=\sum_{\mu} \gamma_{\lambda \mu} \mathrm{i}\left(\boldsymbol{E} \times \boldsymbol{E}^{*}\right)_{\mu}=E_{0}^{2} t_{p} t_{s} P_{\text {circ }}(\boldsymbol{\gamma} \hat{\boldsymbol{e}})_{\lambda},
$$

where $\hat{\boldsymbol{e}}$ is the light propagation unit vector and $\boldsymbol{\gamma}$ is the second-rank pseudotensor. In the systems of $\mathrm{C}_{3 v}$ symmetry, the tensor $\gamma$ has one linearly independent component, namely, $\gamma_{x y}=-\gamma_{y x} \equiv \gamma$. Thus, the CPGE current flows always perpendicularly to the incidence plane.

In our experimental setup, a quarter-wave plate is rotated by the angle $\varphi_{p}$ between the laser light polarization vector $\boldsymbol{E}_{l}$ and the principal axis of the polarizer (see inset to Fig. 5). The total photogalvanic current is given by both second and fourth harmonics of this angle. While radiation handedness is solely determined by the angle $\varphi_{p}$ and does not depend on the relative orientation between $\boldsymbol{E}_{l}$ and the plane of incidence, the orientation of the ellipse is substantially different. In particular, for $\boldsymbol{E}_{l}$ perpendicular to the incidence plane at $\varphi_{p}=0$ ( $s$ polarization) and for $\boldsymbol{E}_{l}$ parallel to the incidence plane at $\varphi_{p}=0$ ( $p$ polarization $)$, the orientation of the corresponding ellipses on the sample differs by $90^{\circ}$. Therefore, in the former case, light is never $p$ polarized, and in the latter case, $s$ polarization cannot be achieved, which is quite different from the geometry of half-wave plate where all states of linear polarization can be obtained. Thus, we derive two sets of equations describing experimental setup of Figs. $5(\mathrm{a})-5(\mathrm{~d})$.

In the first geometry ( $s$ polarization at $\left.\varphi_{p}=0\right)$ from Eqs. (4) and (8), we get

$$
\begin{gathered}
j_{x}\left(\varphi_{p}\right)=\frac{\chi}{2} E_{0}^{2} t_{p}^{2} \cos \theta \sin \theta\left(1-\cos 4 \varphi_{p}\right), \\
j_{y}\left(\varphi_{p}\right)=E_{0}^{2} t_{p} t_{s} \sin \theta\left(-\frac{\chi}{2} \sin 4 \varphi_{p}+\gamma \sin 2 \varphi_{p}\right),
\end{gathered}
$$

and for the latter geometry ( $p$ polarization at $\left.\varphi_{p}=0\right)$,

$$
\begin{gathered}
j_{x}\left(\varphi_{p}\right)=\frac{\chi}{2} E_{0}^{2} t_{p}^{2} \cos \theta \sin \theta\left(3+\cos 4 \varphi_{p}\right), \\
j_{y}\left(\varphi_{p}\right)=E_{0}^{2} t_{p} t_{s} \sin \theta\left(\frac{\chi}{2} \sin 4 \varphi_{p}+\gamma \sin 2 \varphi_{p}\right) .
\end{gathered}
$$

Here, we again took into account that in our samples $\left|\chi^{\prime}\right|$ $\ll|\chi|$. Fits of experiment with the constant $\chi$ independently obtained from experiments with half-wave plates and $\gamma$ as a single adjustable parameter being the same for all curves yields a good agreement with the experiment. Here, again for the longitudinal photocurrent, the offset due to photon drag effect shows up and has the same value as that in the experiments with half-wave plates.

\section{MICROSCOPIC THEORY}

In order to describe the measured dependences of the photocurrent on wavelength and temperature, we have developed a microscopic theory of LPGE. First we consider the contribution proportional to the coefficient $\chi$ in Eq. (4). This contribution does exist not only in systems of the $\mathrm{C}_{3 v}$ or $\mathrm{C}_{6 v}$ symmetry but is also allowed by the uniaxial point group $\mathrm{C}_{\infty v}$. Therefore, we can calculate the $\chi$ contribution to $j_{x, y}^{\mathrm{LPGE}}$ in the uniaxial approximation. Then we turn to calculation of the contribution proportional to $\chi^{\prime}$ in Eq. (4) and surviving under normal incidence due to the reduced $\mathrm{C}_{3 v}$ symmetry of the system. We note that so far the LPGE in 2D systems has been treated theoretically for direct intersubband transitions only. ${ }^{25,26}$ The mechanism of the LPGE under Drude absorption differs strongly because scattering is unavoidably present in the intrasubband absorption process. 
$\mathrm{a}_{1}$

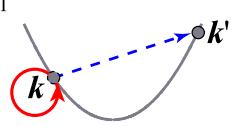

$\mathrm{a}_{2}$

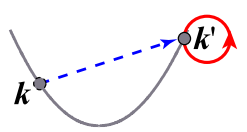

$b_{1}$

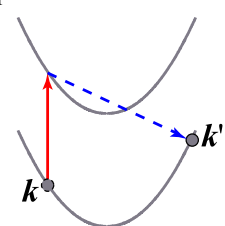

$\mathrm{b}_{2}$

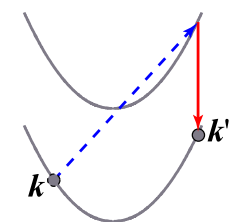

FIG. 7. (Color online) The quantum transitions responsible for the shift contribution to the photocurrent. Note that Drude absorption under oblique light incidence is caused by $a_{1}$ and $a_{2}$ processes only.

\section{A. Microscopic model for oblique incidence}

Microscopically the LPGE current consists of the shift and ballistic contributions, which in general can be comparable in order of magnitude. We start from the analysis of the shift contribution and then turn to the ballistic contribution.

\section{Shift contribution}

The shift contribution arises due to coordinate shifts of the free-carrier wave-packet center-of-mass by microscopic lengths in quantum transitions. The corresponding current is a product of the elementary charge $e$, the transition probability rate $W_{\boldsymbol{k}^{\prime} \boldsymbol{k}}$, and the shift vector $\boldsymbol{R}_{\boldsymbol{k}^{\prime} \boldsymbol{k}}$ or, explicitly,

$$
\boldsymbol{j}_{\mathrm{LPGE}}^{\text {(shift) }}=2 e \sum_{\boldsymbol{k}^{\prime} k} W_{\boldsymbol{k}^{\prime} k} \boldsymbol{R}_{\boldsymbol{k}^{\prime} \boldsymbol{k}}
$$

where the factor "two" accounts for spin degeneracy. The elementary shift is related to the transition matrix element $M_{k^{\prime} k}$ by $^{5}$

$$
\boldsymbol{R}_{\boldsymbol{k}^{\prime} \boldsymbol{k}}=-\frac{\operatorname{Im}\left[M_{\boldsymbol{k}^{\prime} \boldsymbol{k}}^{*}\left(\nabla_{\boldsymbol{k}}+\nabla_{\boldsymbol{k}^{\prime}}\right) M_{\boldsymbol{k}^{\prime} \boldsymbol{k}}\right]}{\left|M_{\boldsymbol{k}^{\prime} \boldsymbol{k}}\right|^{2}}=-\left(\nabla_{\boldsymbol{k}}+\nabla_{\boldsymbol{k}^{\prime}}\right) \arg \left(M_{\boldsymbol{k}^{\prime} \boldsymbol{k}}\right),
$$

where $\arg (u)$ is the phase of the complex number $u$. The above two equations are general and valid for the shift photocurrent calculation in any frequency range. ${ }^{3,4}$ Here, for the first time, we apply them to consider the LPGE under the Drude absorption. Note that Eq. (12) was applied in the theory of anomalous Hall effect ${ }^{6,27}$ where the coordinate shift at scattering is called a side jump.

The main contribution to the matrix element for indirect intrasubband optical transitions,

$$
M_{1 k^{\prime}, 1 k}^{\|}=U_{1 k^{\prime}, 1 k}\left(\frac{V_{k^{\prime}}}{E_{k^{\prime}}-E_{k}}-\frac{V_{k}}{\hbar \omega}\right),
$$

comes from two-quantum processes $a_{1}$ and $a_{2}$ with intermediate virtual states in the same $e 1$ subband (see Fig. 7).

Here, the 2D electron kinetic energy $E_{k}=\hbar^{2} k^{2} / 2 m, m$ is the electron in-plane effective mass, $V_{k}$ is the matrix element of electron-light interaction,

$$
V_{k}=-\frac{e \hbar A}{m c} \boldsymbol{e} \cdot \boldsymbol{k}
$$

$\omega, A$, and $\boldsymbol{e}$ are the frequency, amplitude, and unit polarization vector of the light wave, $U_{1 k^{\prime}, 1 k}$ is the matrix element of intrasubband elastic scattering $e 1, \boldsymbol{k} \rightarrow e 1, \boldsymbol{k}^{\prime}$, and the superscript $\|$ indicates that the process is allowed for the polarization $\boldsymbol{e}$ containing an in-plane component $\boldsymbol{e}_{\|}$. In the following, for simplicity, we ignore the wave vector dependence of $U_{1 k^{\prime}, 1 k}$ and replace it by a constant $U_{11}$. The probability rate $W_{\boldsymbol{k}^{\prime} \boldsymbol{k}}$ is given by Fermi golden rule and expressed via the squared modulus of the transition matrix element and $\delta$ function describing the energy conservation,

$$
E_{k^{\prime}}-E_{k}=\hbar \omega \text {. }
$$

The processes $a_{1}$ and $a_{2}$ in isolation from other processes make no contribution to $j_{\mathrm{LPGE}}^{\text {(shift) }}$ because $M_{1 k^{\prime}, 1 k}^{\|}$is independent of $e_{z}$, while the $\chi$-related photocurrent in the phenomenological Eq. (4) is proportional to $e_{z}$ or because the microscopic expression in the square brackets in Eq. (12) is real and hence the elementary shift is absent. The shift contribution becomes nonzero if two other indirect processes, $b_{1}$ and $b_{2}$ in Fig. 7, are taken into account. The virtual states in these processes lie in the second size-quantized subband $e 2$. The corresponding matrix element reads as

$$
M_{1 k^{\prime}, 1 k}^{\perp}=-\mathrm{i} e_{z} \frac{e A}{\hbar c} z_{21} \Delta_{21}\left(\frac{U_{12}}{\Delta_{21}-\hbar \omega}-\frac{U_{21}}{\Delta_{21}+E_{k^{\prime}}-E_{k}}\right) .
$$

Here, $U_{21}=U_{12}$ is the intersubband scattering matrix element, $\Delta_{21}$ is the energy spacing between the $e 2$ and $e 1$ subbands, and $z_{21}$ is the intersubband matrix element of the coordinate $z$. The superscript $\perp$ indicates that transitions (15) are allowed in the polarization perpendicular to the interface plane.

Substituting $M_{k^{\prime} k}=M_{k^{\prime} k}^{\|}+M_{k^{\prime} k}^{\perp}$ into Eq. (12), taking then into account the energy conservation equation (14) and assuming $\hbar \omega \ll \Delta_{21}$, we obtain for the elementary shift,

$$
\left|M_{\boldsymbol{k}^{\prime} \boldsymbol{k}}\right|^{2} \boldsymbol{R}_{\boldsymbol{k}^{\prime} \boldsymbol{k}}=-z_{21}\left(\frac{e \hbar A}{m c}\right)^{2} \frac{U_{11} U_{21}}{\Delta_{21} \hbar \omega} e_{z}\left[\boldsymbol{e} \cdot\left(3 \boldsymbol{k}^{\prime}-\boldsymbol{k}\right)\right]\left(\boldsymbol{k}^{\prime}-\boldsymbol{k}\right) .
$$

Note that the shift is different from zero only due to the wave vector dependence of denominators $E_{k^{\prime}}-E_{k}$ and $\Delta_{21}+E_{k^{\prime}}$ $-E_{k}$ in the matrix elements (13) and (15).

The result for the shift photocurrent can be presented in the form

$$
\boldsymbol{j}_{\mathrm{LPGE}}^{\text {(shift) }}=-e \xi z_{21} \boldsymbol{e}_{\|} e_{z} \frac{\eta_{\|} I}{\Delta_{21}} \frac{F[4 E+3 \hbar \omega]}{F[2 E+\hbar \omega]} .
$$

Here, the factor $\xi$ is given by ${ }^{28}$

$$
\xi=\frac{\left\langle U_{11} U_{21}\right\rangle}{\left\langle U_{11}^{2}\right\rangle},
$$

where the angular brackets mean averaging over the distribution of static scatterers along $z$, the functional $F[\phi(E)]$ 

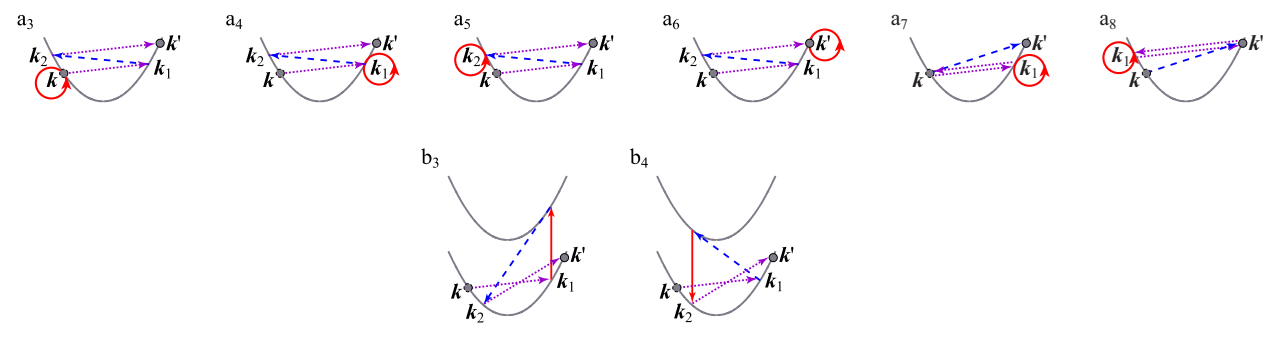

FIG. 8. (Color online) Optical two-impurity-mediated processes included into consideration in order to obtain nonvanishing antisym-

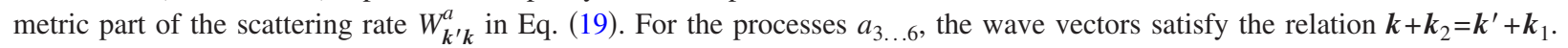

turns any function $\phi(E)$ into a real number as follows:

$$
F[\phi(E)]=\int_{0}^{\infty}[f(E)-f(E+\hbar \omega)] \phi(E) d E / \int_{0}^{\infty} f(E) d E,
$$

$f(E)$ is the Fermi-Dirac distribution function, $\eta_{\|}$is the absorbance under normal incidence $(\theta=0)$ given by ${ }^{29}$

$$
\eta_{\|}=\frac{e^{2}}{\hbar c} \frac{2 \pi N_{s}}{n_{\omega} m \omega^{3} \tau_{p}} F[2 E+\hbar \omega],
$$

the momentum relaxation time $\tau_{p}=\hbar^{3} /\left(m N_{d}\left\langle U_{11}^{2}\right\rangle\right)$, and $N_{s}$ and $N_{d}$ are the $2 \mathrm{D}$ concentrations of electrons and scatterers, respectively.

Note that allowance for nonzero value of $\xi$ and, hence, of the LPGE current, presupposes the uniaxial asymmetry of the structure. ${ }^{28}$ This corresponds to the phenomenology of the LPGE, which requires the absence of an inversion center in the system. A value of $\xi$ is nonzero due to an asymmetrical shape of the electron envelope functions in the $e 1$ and $e 2$ subbands caused by the build-in electric field. An inhomogeneous asymmetrical distribution of scatterers along the $z$ axis can also contribute to $\xi$.

\section{Ballistic contribution}

In order to obtain a nonzero ballistic photocurrent, it is necessary to go beyond the approximation of single-impurity scattering and take into account simultaneous scattering by two impurities in one process. Such optical-absorption processes are schematically shown in Fig. 8. Let us introduce the antisymmetrical part $W_{\boldsymbol{k}^{\prime} \boldsymbol{k}}^{a}$ of the $\boldsymbol{k} \rightarrow \boldsymbol{k}^{\prime}$ transition rate $W_{k^{\prime} k}$, which changes sign under simultaneous inversion of the wave vectors $\boldsymbol{k}$ and $\boldsymbol{k}^{\prime}$. It can be expressed in terms of Fermi golden rule,

$$
W_{k^{\prime} k}^{a}=\frac{2 \pi}{\hbar}\left|M_{k^{\prime} k}\right|_{a}^{2}\left[f\left(E_{k}\right)-f\left(E_{k^{\prime}}\right)\right] \delta\left(E_{k^{\prime}}-E_{k}-\hbar \omega\right),
$$

where $\left|M_{k^{\prime}}\right|_{a}^{2}$ is the antisymmetric part of the squared modulus of the optical two-impurity-mediated matrix element $M_{k^{\prime} k}$. Then the ballistic contribution to the LPGE current is given by

$$
\boldsymbol{j}_{\mathrm{LPGE}}^{\text {(ball) }}=2 e \sum_{\boldsymbol{k}, \boldsymbol{k}^{\prime}} W_{\boldsymbol{k}^{\prime} \boldsymbol{k}}^{a} \tau_{p}\left(\boldsymbol{v}_{\boldsymbol{k}^{\prime}}-\boldsymbol{v}_{\boldsymbol{k}}\right)
$$

where $\boldsymbol{v}_{\boldsymbol{k}}=\hbar \boldsymbol{k} / \mathrm{m}$ is the electron velocity.

Here it is worth discussing the dependence of the ballistic and shift contributions on the concentration of elastic scatterers $N_{d}$. For two-impurity scattering the probability rate $W_{\boldsymbol{k}^{\prime} \boldsymbol{k}}^{a} \propto N_{d^{\prime}}^{2}$. In contrast, the single-impurity scattering rate $W_{\boldsymbol{k}^{\prime} \boldsymbol{k}}$ in Eq. (11) linearly depends on $N_{d}$. However, the ballistic current is controlled by the momentum relaxation time [see the factor $\tau_{p}$ in the right-hand side of Eq. (20)]. Since $\tau_{p} \propto N_{d}^{-1}$, the ballistic and shift currents are both proportional to $N_{d}$. In this sense, there is an analogy between a pair of the two contributions to the photocurrent and a pair of the sidejump and skew-scattering currents in the anomalous Hall effect. $^{6}$

In order to get the photocurrent proportional to both $\boldsymbol{e}_{\|}$ and $e_{z}$, we extract from $\left|M_{k^{\prime} k}\right|^{2}$ the interference term proportional to $\operatorname{Re}\left\{M^{\|} M^{\perp *}\right\}$, where $M^{\|}$and $M^{\perp}$ are the contributions to the matrix element of the indirect optical transition proportional to $e_{x, y}$ and $e_{z}$, respectively. To the lowest order in $\hbar \omega / \Delta_{21} \ll 1$, one should include into consideration six two-impurity scattering processes $a_{3} \cdots a_{8}$ contributing to $M^{\|}$ and two processes $b_{3}$ and $b_{4}$ for $M^{\perp}$ depicted in Fig. 8 .

For example, below we present the contributions to $M^{\|}$ from the processes $a_{5}$ and $a_{6}$,

$$
\begin{gathered}
M_{a_{5}}^{\|}=N_{d} \sum_{\boldsymbol{k}_{1} \boldsymbol{k}_{2}} \frac{U_{11}^{\prime} V_{\boldsymbol{k}_{2}} U_{11} U_{11}^{\prime} \delta_{\boldsymbol{k}+\boldsymbol{k}_{2}, \boldsymbol{k}^{\prime}+\boldsymbol{k}_{1}}}{\left(E_{\boldsymbol{k}_{2}}-E_{\boldsymbol{k}}-\hbar \omega-\mathrm{i} 0\right)\left(E_{\boldsymbol{k}_{2}}-E_{\boldsymbol{k}}-\mathrm{i} 0\right)\left(E_{\boldsymbol{k}_{1}}-E_{\boldsymbol{k}}-\mathrm{i} 0\right)}, \\
M_{a_{6}}^{\|}=N_{d} \sum_{\boldsymbol{k}_{1} \boldsymbol{k}_{2}} \frac{V_{\boldsymbol{k}^{\prime}} U_{11}^{\prime} U_{11} U_{11}^{\prime} \delta_{\boldsymbol{k}+\boldsymbol{k}_{2}, \boldsymbol{k}^{\prime}+\boldsymbol{k}_{1}}}{\left(E_{\boldsymbol{k}^{\prime}}-E_{\boldsymbol{k}}-\mathrm{i} 0\right)\left(E_{\boldsymbol{k}_{2}}-E_{\boldsymbol{k}}-\mathrm{i} 0\right)\left(E_{\boldsymbol{k}_{1}}-E_{\boldsymbol{k}}-\mathrm{i} 0\right)},
\end{gathered}
$$


where the prime means the scattering (shown by dotted lines in Fig. 8) by a defect different from that which is related to the matrix element $U_{11}$ in these equations and to $U_{21}$ in Eq. (15) (shown by the dashed lines). The Kronecker symbols are obtained after averaging the matrix element over the inplane distribution of the second scatterer. Note that averaging over its distribution in the $z$ direction results in replacement of the square $\left(U_{11}^{\prime}\right)^{2}$ by $\left\langle U_{11}^{2}\right\rangle$. The energy denominators are rewritten by using the identity

$$
\frac{1}{x-\mathrm{i} 0}=P V \frac{1}{x}+\mathrm{i} \pi \delta(x),
$$

and, among three such terms in each contribution $M_{a_{j}}^{\|}(j$ $=3 \ldots 8)$, we retain the $\delta$ function in one of them and take the Cauchy principal values for two others. This additional $\delta$ function, together with the energy conservation law (14) and the relation $\boldsymbol{k}+\boldsymbol{k}_{2}=\boldsymbol{k}^{\prime}+\boldsymbol{k}_{1}$, fixes absolute values of the wave vectors $\boldsymbol{k}, \boldsymbol{k}^{\prime}, \boldsymbol{k}_{1}$, and $\boldsymbol{k}_{2}$. The integration over the azimuthal angle of the vector $\boldsymbol{k}_{1}$ or $\boldsymbol{k}_{2}$ is performed by using the principal value integral,

$$
P V \int_{0}^{2 \pi} \frac{d \varphi}{2 \pi} \frac{1}{a-b \cos \varphi}=\frac{\Theta\left(a^{2}-b^{2}\right) \operatorname{sign} a}{\sqrt{a^{2}-b^{2}}},
$$

where $\Theta(x)$ is the Heaviside step function. The calculation shows that, among the eight processes presented in Fig. 8, only two, namely, the processes $a_{5}$ and $a_{6}$, lead to nonzero ballistic current. The final result for this current reads

$$
j_{\mathrm{LPGE}}^{\text {(ball) }}=\boldsymbol{e}_{\|} e_{z} \frac{\eta_{\|} I}{\Delta_{21}} \xi e z_{21} \frac{F[L(\hbar \omega / E)]}{F[E / \hbar \omega+1 / 2]},
$$

where

$$
\begin{gathered}
L(x)=\frac{x}{2 \pi} \int_{\varphi_{0}(x)}^{2 \pi} \frac{d \varphi}{\sqrt{(x-2 \sqrt{1+x} \cos \varphi)^{2}-4}}, \\
\varphi_{0}(x)=\Theta(8-x) \arccos \left(\frac{x-2}{2 \sqrt{1+x}}\right) .
\end{gathered}
$$

The function $L(x)$ goes to zero as $x / 4$ at $x \rightarrow 0$ and tends to 1 at $x \rightarrow \infty$. A sum of the shift and ballistic contributions given by Eqs. (17) and (22) represents the photocurrent phenomenologically described by the coefficient $\chi$ in Eq. (4).

\section{B. Microscopic model for normal incidence}

An ideal GaN/AlN interface has the point-group symmetry $C_{3 v}$, which is a subgroup of the $C_{6 v}^{4}$ space group of a bulk wurtzite crystal. The group $C_{6 v}^{4}$ is nonsymmorphic and contains, in addition to $C_{3}$ and $\sigma_{v}$, the elements $\left\{C_{6} \mid \boldsymbol{\tau}\right\},\left\{\sigma_{d} \mid \boldsymbol{\tau}\right\}$, where $\boldsymbol{\tau}$ is the fractional lattice translation $(0,0, c / 2)$ and $c$ is the lattice constant along the principal axis $z$. In the $2 \mathrm{D}$ structures, the screw-axis and glide-plane operations shift the interfaces along $z$ not bringing the system into coincidence with itself and, as a result, the point-group symmetry reduces to $C_{3 v}$.

For the $C_{6 v}$ symmetry of bulk wurtzite structure, characteristic of III-nitrides, the nonvanishing third order tensor components $\chi_{\lambda \mu \nu}$ symmetrical in $\mu$ and $\nu$ are $\chi_{z z z}, \chi_{z x x}$ $=\chi_{z y y}$, and $\chi_{x x z}=\chi_{x z x}=\chi_{y y z}=\chi_{y z y} \equiv \chi$. Therefore, forbidden are such bulk effects as the second-harmonic generation for excitation along $z$, a piezoelectric field induced in the $(x, y)$ plane under the deformation in this plane and the photocurrent in the $(x, y)$ plane for the light propagating along $z$. The reduction from $C_{6 v}$ to $C_{3 v}$ removes these restrictions and four new nonzero components appear, $\chi_{x x y}=\chi_{x y x}=\chi_{y x x}=-\chi_{y y y}$ $\equiv \chi^{\prime}$ [see Eqs. (4)]. Note that it is the interface that is exclusively responsible for the appearance of new components.

Here we propose a microscopic model of the LPGE under normal incidence. In this geometry virtual transitions via other size-quantized electronic subbands are forbidden; therefore, we should consider the light absorption with intermediate states either in the same $e 1$ electronic subband or in the valence band. In the first case, see Fig. 7 , the reduced $C_{3 v}$ symmetry manifests itself in the scattering matrix element $U_{1 k^{\prime}, 1 k}$, and, instead of Eq. (16), one should replace the elementary shift in the product $\left|M_{\boldsymbol{k}^{\prime} \boldsymbol{k}}\right|^{2} \boldsymbol{R}_{\boldsymbol{k}^{\prime} \boldsymbol{k}}$ by the scattering induced shift,

$$
\boldsymbol{R}_{\boldsymbol{k}^{\prime} \boldsymbol{k}}^{(U)}=-\frac{\operatorname{Im}\left[U_{1 \boldsymbol{k}^{\prime}, 1 \boldsymbol{k}}^{*}\left(\nabla_{\boldsymbol{k}}+\nabla_{\boldsymbol{k}^{\prime}}\right) U_{1 \boldsymbol{k}^{\prime}, 1 \boldsymbol{k}}\right]}{\left|U_{1 \boldsymbol{k}^{\prime}, 1 \boldsymbol{k}}\right|^{2}} .
$$

Note that the main contribution to $U_{1 k^{\prime}, 1 k}$ dependent only on the difference $\left(\boldsymbol{k}^{\prime}-\boldsymbol{k}\right)$ does not result in the shift because the operator $\left(\nabla_{k}+\nabla_{k^{\prime}}\right)$ nullifies such a term. Similar contribution to the LPGE current has been calculated for Drude absorption in the heavy-hole subband of a bulk $p$-GaAs, ${ }^{30}$ where the shift appeared due to interference of polar and deformation scattering mechanisms on optical phonons.

For optical transitions going via $\Gamma_{6}$ valence-band states, the elementary shift can be related to asymmetry of the interband optical matrix elements, in which case the asymmetry of the interband scattering matrix element is neglected. We consider this mechanism in more detail. Taking into account the symmetry considerations, we can present the interband matrix elements of the operator $\boldsymbol{e}_{\|} \cdot \boldsymbol{p}$ (where $\boldsymbol{p}$ is the momentum operator) between the conduction-band state $\Gamma_{1}$ and the valence-band states $\Gamma_{6}$ as

$$
\begin{gathered}
\boldsymbol{e}_{\|} \cdot \boldsymbol{p}_{c \Gamma_{1} ; v, \Gamma_{6 x}}(\boldsymbol{k})=i \mathcal{P} e_{x}+\mathcal{Q}\left(e_{x} k_{y}+e_{y} k_{x}\right), \\
\boldsymbol{e} \cdot \boldsymbol{p}_{c \Gamma_{1} ; v, \Gamma_{6 y}}(\boldsymbol{k})=i \mathcal{P} e_{y}+\mathcal{Q}\left(e_{x} k_{x}-e_{y} k_{y}\right),
\end{gathered}
$$

where $\mathcal{P}$ and $\mathcal{Q}$ are real constants. Multiband derivation of these contributions for GaN-based 2D structures is given in Appendix B. The matrix element $\mathcal{Q}$ is nonzero due to the presence of the interface in the heterostructure. This approach is similar to description of interface inversion asymmetry induced spin splitting in the envelope-function method, where the $\delta$-functional terms are introduced into the interband Hamiltonian (see Refs. 31 and 32). As a result, we expect a strong dependence of $\mathcal{Q}$ on the confinement size because $\mathcal{Q}$ is proportional to a product of the electron and hole envelope functions at the interface [see Eq. (B3)]. We expand $M_{\boldsymbol{k}^{\prime} \boldsymbol{k}}$ and $\boldsymbol{R}_{\boldsymbol{k}^{\prime} \boldsymbol{k}}$ in powers of small parameters $\hbar \omega / E_{g}$, $\bar{E} / E_{g} \ll 1$. In the same nonvanishing order, we should take into account $k$ dependence of the matrix element $\mathcal{P}$. This 
could be done by replacing in Eq. (23) the constant $\mathcal{P}$ by the function

$$
\mathcal{P}(\boldsymbol{k})=\mathcal{P}_{0}\left(1-\frac{E_{\boldsymbol{k}}}{2 E_{g}}\right) .
$$

The interband scatterings $c \Gamma_{1} \boldsymbol{k} \leftrightarrow v \Gamma_{6 x} \boldsymbol{k}^{\prime}$ and $c \Gamma_{1} \boldsymbol{k} \leftrightarrow v \Gamma_{6 y} \boldsymbol{k}^{\prime}$ are described by the constant matrix elements $U_{c v_{x}}$ and $U_{c v_{y}}$ assumed to be independent of $\boldsymbol{k}$ and $\boldsymbol{k}^{\prime}$, with $\left\langle U_{c v_{x}}^{2 x}\right\rangle=\left\langle U_{c v_{y}}^{2}\right\rangle^{y}$.

In order to carry out a reasonable estimation of the coefficient $\chi^{\prime}$ in Eq. (4), we have used a simple nonrelativistic $\boldsymbol{k} \cdot \boldsymbol{p}$ model coupling the $c \Gamma_{1}$ and $v \Gamma_{6}$ band states. In this model the valence band consists of two subbands, one with an infinite in-plane effective mass and the other with the hole in-plane effective mass coinciding with that for a conduction-band electron. Omitting the details of derivation, we present the final result,

$$
\chi^{\prime}=\frac{2 \pi e^{3}}{m_{0}^{2} \omega} \frac{\left\langle U_{c v_{x}}^{2}\right\rangle \mathcal{P}_{0} \mathcal{Q}}{E_{g}^{3}} N_{s} F_{1},
$$

where $F_{1} \equiv F[1]=\int_{0}^{\infty}[f(E)-f(E+\hbar \omega)] d E / \int_{0}^{\infty} f(E) d E$. Experiment shows a noticeable magnitude of the LPGE current under normal incidence (see Fig. 4).

\section{DISCUSSION}

In this section, we discuss the role of the contributions to the total current and analyze the frequency and temperature dependences of LPGE. An analytical estimation for the shift and ballistic photocurrents at Drude absorption is readily available for the photon energies $\hbar \omega$ small compared to the electron typical energy $\bar{E}$, which is relevant to our experiments performed at room temperature and applying terahertz radiation. In this case, Eqs. (17) and (22) reduce to

$$
\begin{gathered}
j_{\mathrm{LPGE}}^{\text {(shift) }} \sim 2 \xi e z_{21} \frac{\eta_{\|} I}{\Delta_{21}}, \\
j_{\mathrm{LPGE}}^{\text {(ball) }} \sim \xi e z_{21} \frac{\eta_{\|} I}{\Delta_{21}}\left(\frac{\hbar \omega}{2 \bar{E}}\right)^{2} .
\end{gathered}
$$

Since $\hbar \omega / \bar{E} \ll 1$ the shift contribution dominates the LPGE. This estimation is confirmed by the study of the frequency dependence of LPGE. Indeed, while the shift photocurrent follows the frequency dependence of the absorbance which, for Drude processes, is given by Eq. (1), the ballistic contribution has another frequency dependence because in this case $j_{\text {LPGE }}^{\text {(ball) }} \propto \eta_{\|} \omega^{2}$. The observed good agreement of the current and absorption frequency behavior (see Fig. 3) unambiguously proves the dominating role of the shift contribution to the LPGE. We note that such a spectral behavior of the LPGE has been previously observed in bulk III-V semiconductors. ${ }^{33}$

We note that even for the opposite limit of $\hbar \omega / \bar{E} \gg 1$ the LPGE due to Drude absorption is still dominated by the shift contribution. In this case, estimations yield $\boldsymbol{j}_{\mathrm{LPGE}}^{\text {(shift) }}=-(3 / 2) \boldsymbol{j}_{\mathrm{LPGE}}^{\text {(ball) }}$.

Now we analyze the temperature dependence. As follows from Eq. (17), the temperature dependence of the LPGE cur- rent is given by $j_{\mathrm{LPGE}}^{(\mathrm{shift})} \propto \xi \eta_{\|}(T)$. Taking into account temperature behavior of the Drude absorption [see Eq. (18)], we get $j_{\text {LPGE }}^{\text {(shift })} \propto \xi / \tau_{p}(T)$. Here we again use the relation $\hbar \omega \ll \bar{E}$ $\sim k_{B} T$. Variation of the temperature results in the change of the dominant scattering mechanisms. Typically in the lowdimensional structures by raising temperature, we get a transition from the temperature-independent scattering rate by impurities $1 / \tau_{p}^{\mathrm{jmp}}$ to the fast-increasing rate due to scattering by phonons $1 / \tau_{p}^{\mathrm{ph}}(T)$. As a result, we have for the LPGE current

$$
j_{\text {LPGE }}^{\text {(shift })} \propto \frac{\xi_{\text {imp }}}{\tau_{p}^{\text {jmp }}}+\frac{\xi_{\mathrm{ph}}}{\tau_{p}^{\mathrm{ph}}(T)} .
$$

This expression demonstrates that despite the inverse mobility $1 / \mu(T) \propto 1 / \tau_{p}^{\mathrm{imp}}+1 / \tau_{p}^{\mathrm{ph}}(T)$ is a monotonous function of temperature (see inset in Fig. 3) and the asymmetry factors $\xi$ are temperature independent, the LPGE current can have a minimum or even change the sign at temperature increase. This is due to the interplay between two contributions to the LPGE caused by different scattering mechanisms characterized by substantially different values of $\xi_{\text {imp }}$ and $\xi_{\text {ph }}$ possibly being even opposite in signs. Due to the fact that only one time is temperature dependent, $\left[\tau_{p}^{\mathrm{ph}}(T)\right]$ variation of temperature may result in the dominance of one or the other contribution to the LPGE current.

\section{CONCLUSION}

In conclusion, we have studied the linear photogalvanic effect in wurtzite $\mathrm{GaN}(0001)$-based heterojunctions under excitation by linearly and elliptically polarized lights in the terahertz frequency range. Experimentally, the properties of LPGE have been revealed as a function of the incidence angle and the polarization state of radiation. A detailed pointgroup symmetry analysis of the polarization properties shows an agreement between the experimental data and the phenomenological theory of LPGE. Phenomenologically, the effect is described by the two linearly independent coefficients $\chi$ and $\chi^{\prime}$, the former being allowed by the $C_{3 v}, C_{6 v}$, and even $C_{\infty v}$ symmetries and the latter being nonzero only for the $C_{3 v}$ symmetry. An observation of the photocurrent under normal incidence determined by $\chi^{\prime}$ unambiguously demonstrates a reduction in the heterojunction symmetry from hexagonal $C_{6 v}$ to trigonal $C_{3 v}$. However, beginning from very small oblique angles first contribution to the photocurrent prevails over the second one. The frequency dependence of the measured LPGE photocurrent exhibits a Drudetype behavior. A microscopic theory of LPGE under the intrasubband (Drude) absorption in the lowest $e 1$ electron subband has been developed. While calculating the $\chi$-related photocurrent, we have included virtual indirect optical transitions via the higher subband $e 2$, estimated both the shift and ballistic contributions and showed that the first one exceeds the other by a large factor $\propto(\bar{E} / \hbar \omega)^{2}$. The dominating shift photocurrent repeats a Lorentzian frequency dependence of the Drude-absorption coefficient, in agreement with the experiment. The temperature dependence of the photocurrent is governed by a product of the absorption coefficient and the dimensionless parameter of asymmetry. Microscopically, the contribution nonvanishing under normal incidence 


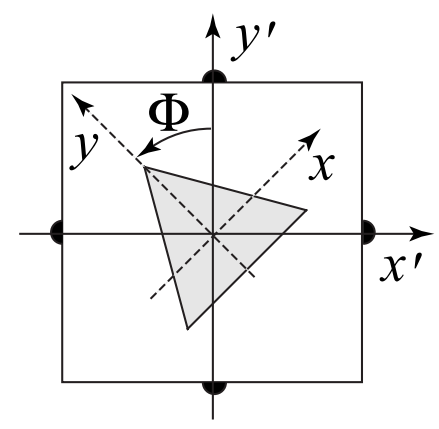

FIG. 9. Schematic representation of the orientation of the crystallographic axes $x$ and $y$ relative to the directions $x^{\prime}$ and $y^{\prime}$ along which the current is measured.

arises taking into account indirect optical transitions via virtual electronic states in the valence band.

\section{ACKNOWLEDGMENTS}

The financial support from the DFG and RFBR is gratefully acknowledged. E.L.I. thanks DFG for the Merkator professorship. Work of L.E.G. is also supported by "Dynasty" Foundation-ICFPM and President grant for young scientists. The high quality GaN samples were kindly provided by Hyun-Ick Cho and Jung-Hee Lee from Kyungpook National University, Korea. We gratefully acknowledge the support of the Stichting voor Fundamenteel Onderzoek der Materie (FOM) in providing beam time on FELIX.

\section{APPENDIX A: PHOTOCURRENTS IN THE ROTATED COORDINATE FRAME}

In Sec. IV A we get phenomenological equations for LPGE at normal incidence. We obtained that the photocurrent direction is determined by the orientation of the electric vector with respect to the crystallographic axes $x$ and $y$. Most convenient for this effect is to investigate the current in the directions along and perpendicular to one of the mirrorreflection planes of the $C_{3 v}$ point group. However, in contrast to III-V semiconductor heterostructures where well determined in-plane crystallographic orientation is naturally obtained by cleaving in GaN/AlGaN heterojunctions grown on the sapphire substrate this may be not the case. Therefore, we obtain here the LPGE for the arbitrary orientation of contacts in respect to the crystallographic directions. To describe this situation, we introduce the angle $\Phi$ between the in-plane directions $x^{\prime}$ and $y^{\prime}$ along, which the contacts are made and the crystallographic axes $x$ and $y$ (see Fig. 9). The symmetry considerations yield the expression for the combination of $x^{\prime}$ and $y^{\prime}$ components of the photocurrent, which follows from Eqs. (4) for $E_{z}=0$,

$$
j_{x^{\prime}}+\mathrm{i} j_{y^{\prime}}=\mathrm{i} \chi^{\prime}\left(E_{x^{\prime}}-\mathrm{i} E_{y^{\prime}}\right)\left(E_{x^{\prime}}^{*}-\mathrm{i} E_{y^{\prime}}^{*}\right) \exp (3 \mathrm{i} \Phi)
$$

or, equivalently,

$$
\begin{aligned}
& j_{x^{\prime}}=\chi^{\prime}\left(t_{0} E_{0}\right)^{2} \sin (2 \alpha-3 \Phi), \\
& j_{y^{\prime}}=\chi^{\prime}\left(t_{0} E_{0}\right)^{2} \cos (2 \alpha-3 \Phi),
\end{aligned}
$$

where $\alpha$ is counted from $y^{\prime}: \boldsymbol{E}(\alpha=0) \| y^{\prime}$.
Equations (A1) demonstrate that the azimuth angular dependence for arbitrary orientation of contacts in respect to the crystallographic directions remains the same but differs by the phase shift $3 \Phi$. Finally, we note that the photocurrent determined by the constant $\chi$ in Eq. (4) is independent of the angle $\Phi$ because it is totally defined by position of the incidence plane and polarization of light. Therefore, Eq. (7) holds for any angle $\Phi$.

\section{APPENDIX B: DERIVATION OF INTERBAND MATRIX ELEMENTS}

Equation (23) can be obtained from the two-band electron effective Hamiltonian

$$
\mathcal{H}_{c \Gamma_{1} ; v, \Gamma_{6 x}}(\boldsymbol{k})=\frac{\hbar}{m_{0}}\left(\mathrm{i} \mathcal{P} k_{x}+\mathcal{Q} k_{x} k_{y}\right),
$$

$$
\mathcal{H}_{c \Gamma_{1} ; v, \Gamma_{6 y}}(\boldsymbol{k})=\frac{\hbar}{m_{0}}\left[\mathrm{i} \mathcal{P} k_{y}+\mathcal{Q} \frac{1}{2}\left(k_{x}^{2}-k_{y}^{2}\right)\right]
$$

by using the relation

$$
\boldsymbol{e} \cdot \boldsymbol{p}_{c \Gamma_{1} ; v, \Gamma_{6}}(\boldsymbol{k})=\frac{m_{0}}{\hbar}\left(\boldsymbol{e} \cdot \nabla_{\boldsymbol{k}}\right) \mathcal{H}_{c \Gamma_{1} ; v, \Gamma_{6}}(\boldsymbol{k}) .
$$

We describe the symmetry reduction from $C_{6 v}$ to $C_{3 v}$ by introducing a $\delta$-functional perturbation $\hat{V}^{\prime} \delta\left(z-z_{\text {if }}\right)$, where $z_{\text {if }}$ is the interface coordinate in the $z$ axis and the operator $\hat{V}^{\prime}$ transforms according to the representation $B_{1}$ of the group $\mathrm{C}_{6 v}$ with the basic function $y^{3}-3 x^{2} y$ (here as before we assume that one of the three mirror-reflection planes in the $C_{3 v}$ group contains the axes $y, z$ and is perpendicular to $x$ ). The perturbation $\hat{V}^{\prime}$ mixes the Bloch state $\Gamma_{6}$ with $\Gamma_{5}$ and the Bloch state $\Gamma_{3}$ with $\Gamma_{1}$.

In order to find the quadratic-in- $\boldsymbol{k}$ correction in Eq. (B1), we calculate the third-order correction to the Hamiltonian using the perturbation theory, ${ }^{34}$

$$
\begin{gathered}
\hat{\mathcal{H}}_{c ; v}^{(3)}=\frac{1}{2} \sum_{s s^{\prime}} \hat{\mathcal{H}}_{c ; s} \hat{\mathcal{H}}_{s, s^{\prime}} \hat{\mathcal{H}}_{s^{\prime} v} \Phi\left(s s^{\prime}\right), \\
\Phi\left(s s^{\prime}\right)=\left[\frac{1}{\left(E_{c}^{0}-E_{s}^{0}\right)\left(E_{c}^{0}-E_{s^{\prime}}^{0}\right)}+\frac{1}{\left(E_{v}^{0}-E_{s}^{0}\right)\left(E_{v}^{0}-E_{s^{\prime}}^{0}\right)}\right] .
\end{gathered}
$$

Here the indices $c, v$ mean, respectively, the lowest conduction and the highest valence bands of the symmetries $\Gamma_{1}$ and $\Gamma_{6} ; s, s^{\prime}$ are other conduction or valence bands different from $c$ and $v$ (the electron spectrum throughout the Brillouin zone for bulk GaN can be found, e.g., in Refs. 35 and 36). $\hat{\mathcal{H}}_{n, n^{\prime}}$ is the matrix of the first-order matrix elements of the $\boldsymbol{k} \cdot \boldsymbol{p}$ coupling or the interface mixing between the states in the bands $n$ and $n^{\prime} ; E_{n}^{0}$ is the electron energy in the band $n$ at the $\Gamma$ point. The dimension of the matrix $\hat{\mathcal{H}}_{n, n^{\prime}}$ is $N \times N^{\prime}$, where $N, N^{\prime}$ are degeneracies of the bands $n$ and $n^{\prime}$, respectively. Taking into account the bands $s \Gamma_{6}(s \neq v), s^{\prime} \Gamma_{5}$, and $s^{\prime \prime} \Gamma_{3}$, we can present the coefficient $\mathcal{Q}$ in Eq. (B1) for a structure with a single interface in the following form: 


$$
\begin{gathered}
\mathcal{Q}=\mathcal{V} \delta\left(z-z_{\mathrm{if}}\right), \\
\mathcal{V}=\frac{\hbar}{m_{0}}\left[P^{\prime}\left(R^{\prime} V_{2}-V_{1} R\right) \Phi\left(s s^{\prime}\right)-V_{3} P^{\prime \prime} R \Phi\left(s^{\prime \prime} s^{\prime}\right)\right] .
\end{gathered}
$$

The set of matrix elements is defined as follows:

$$
\begin{gathered}
V_{1}=\left\langle s, \Gamma_{6 x}\left|\hat{V}^{\prime}\right| s^{\prime}, \Gamma_{5 x}\right\rangle, \quad V_{2}=\left\langle v, \Gamma_{6 x}\left|\hat{V}^{\prime}\right| s^{\prime}, \Gamma_{5 x}\right\rangle, \\
V_{3}=\left\langle c, \Gamma_{1}\left|\hat{V}^{\prime}\right| s^{\prime \prime}, \Gamma_{3}\right\rangle, \\
P^{\prime}=-\mathrm{i}\left\langle c, \Gamma_{1}\left|p_{x}\right| s, \Gamma_{6 x}\right\rangle, \quad P^{\prime \prime}=-\mathrm{i}\left\langle s^{\prime \prime}, \Gamma_{3}\left|p_{x}\right| s^{\prime}, \Gamma_{5 x}\right\rangle,
\end{gathered}
$$

$$
R=-\mathrm{i}\left\langle s^{\prime}, \Gamma_{5 x}\left|p_{x}\right| v, \Gamma_{6 x}\right\rangle, \quad R^{\prime}=-\mathrm{i}\left\langle s^{\prime}, \Gamma_{5 x}\left|p_{x}\right| s, \Gamma_{6 x}\right\rangle .
$$

For the band $s^{\prime} \Gamma_{5}$, we choose the basis $\left|s, \Gamma_{5 x}\right\rangle$ and $\left|s, \Gamma_{5 y}\right\rangle$, which transforms under operations of the $\mathrm{C}_{3 v}$ group as the coordinates $x$ and $y$, i.e., as functions $\left|s^{\prime}, \Gamma_{6 x}\right\rangle,\left|s^{\prime}, \Gamma_{6 y}\right\rangle$. While deriving Eq. (B2) we took into account the relation between matrix elements imposed by the symmetry, e.g., $\left\langle c, \Gamma_{1}\left|p_{y}\right| s, \Gamma_{6 y}\right\rangle=\left\langle c, \Gamma_{1}\left|p_{x}\right| s, \Gamma_{6 x}\right\rangle, \quad\left\langle c, \Gamma_{5 y}\left|p_{x}\right| s, \Gamma_{6 x}\right\rangle=$ $-\left\langle c, \Gamma_{5 y}\left|p_{y}\right| s, \Gamma_{6 y}\right\rangle=\left\langle c, \Gamma_{5 x}\left|p_{x}\right| s, \Gamma_{6 x}\right\rangle$, etc.

It follows then that, for a heterojunction, the interband optical matrix elements are indeed given by Eq. (23) where

$$
\mathcal{P}=P \int f_{e}(z) f_{h}(z) d z, \quad \mathcal{Q}=\mathcal{V} f_{e}\left(z_{\mathrm{if}}\right) f_{h}\left(z_{\mathrm{if}}\right),
$$

$P=-\mathrm{i}\left\langle c, \Gamma_{1}\left|p_{x}\right| v, \Gamma_{6 x}\right\rangle=-\mathrm{i}\left\langle c, \Gamma_{1}\left|p_{y}\right| v, \Gamma_{6 y}\right\rangle$, and $f_{e}(z)$ and $f_{h}(z)$ are the electron and hole envelope functions. *sergey.ganichev@physik.uni-regensburg.de

${ }^{1}$ H. Linke, Appl. Phys. A: Mater. Sci. Process. 75, 167 (2002).

${ }^{2}$ A. M. Glass, D. von der Linde, and T. J. Negran, Appl. Phys. Lett. 25, 233 (1974).

${ }^{3}$ B. I. Sturman and V. M. Fridkin, The Photovoltaic and Photorefractive Effects in Noncentrosymmetric Materials (Gordon and Breach, Philadelphia, 1992).

${ }^{4}$ E. L. Ivchenko, Optical Spectroscopy of Semiconductor Nanostructures (Alpha Science International, Harrow, UK, 2005).

${ }^{5}$ V. I. Belinicher, E. L. Ivchenko, and B. I. Sturman, Zh. Eksp. Teor. Fiz. 83, 649 (1982) [Sov. Phys. JETP 56, 359 (1982)].

${ }^{6}$ N. A. Sinitsyn, J. Phys.: Condens. Matter 20, 023201 (2008).

${ }^{7}$ W. Weber, S. D. Ganichev, Z. D. Kvon, V. V. Bel'kov, L. E. Golub, S. N. Danilov, D. Weiss, W. Prettl, H.-I. Cho, and J.-H. Lee, Appl. Phys. Lett. 87, 262106 (2005).

${ }^{8}$ W. Weber, S. D. Ganichev, S. Seidl, V. V. Bel'kov, L. E. Golub, W. Prettl, Z. D. Kvon, Hyun-Ick Cho, and Jung-Hee Lee, in Physics of Semiconductors: 28th International Conference on the Physics of Semiconductors - ICPS 2006, AIP Conf. Proc. No. 893 (AIP, Melville, NY, 2007), p. 1311.

${ }^{9}$ X. W. He, B. Shen, Y. Q. Tang, N. Tang, C. M. Yin, F. J. Xu, Z. J. Yang, G. Y. Zhang, Y. H. Chen, C. G. Tang, and Z. G. Wang, Appl. Phys. Lett. 91, 071912 (2007).

${ }^{10}$ Y. Q. Tang, B. Shen, X. W. He, K. Han, N. Tang, W. H. Chen, Z. J. Yang, G. Y. Zhang, Y. H. Chen, C. G. Tang, Z. G. Wang, K. S. Cho, and Y. F. Chen, Appl. Phys. Lett. 91, 071920 (2007).

${ }^{11}$ K. S. Cho, C.-T. Liang, Y. F. Chen, Y. Q. Tang, and B. Shen, Phys. Rev. B 75, 085327 (2007).

${ }^{12}$ W. Weber, S. Seidl, V. V. Bel'kov, L. E. Golub, E. L. Ivchenko, W. Prettl, Z. D. Kvon, Hyun-Ick Cho, Jung-Hee Lee, and S. D. Ganichev, Solid State Commun. 145, 56 (2008).

${ }^{13}$ S. Nakamura and G. Fasol, The Blue Laser Diode, GaN Based Light Emitters and Lasers (Springer, Berlin, 1997).

${ }^{14} \mathrm{~S}$. D. Ganichev and W. Prettl, Intense Terahertz Excitation of Semiconductors (Oxford University Press, Oxford, 2006).

${ }^{15}$ G. M. H. Knippels, X. Yan, A. M. MacLeod, W. A. Gillespie, M. Yasumoto, D. Oepts, and A. F. G. van der Meer, Phys. Rev. Lett. 83, 1578 (1999).

${ }^{16}$ K. Seeger, Semiconductor Physics (Springer, Wien, 1997).

${ }^{17}$ N. V. Smith, Phys. Rev. B 64, 155106 (2001).

${ }^{18}$ K. S. Cho, Tsai-Yu Huang, Hong-Syuan Wang, Ming-Gu Lin, Tse-Ming Chen, C.-T. Liang, Y. F. Chen, and Ikai Lo, Appl.
Phys. Lett. 86, 222102 (2005).

${ }^{19}$ N. Tang, B. Shen, M. J. Wang, K. Han, Z. J. Yang, K. Xu, G. Y. Zhang, T. Lin, B. Zhu, W. Z. Zhou, and J. H. Chu, Appl. Phys. Lett. 88, 172112 (2006).

${ }^{20}$ N. Thillosen, Th. Schäpers, N. Kaluza, H. Hardtdegen, and V. A. Guzenko, Appl. Phys. Lett. 88, 022111 (2006).

${ }^{21}$ S. Schmult, M. J. Manfra, A. Punnoose, A. M. Sergent, K. W. Baldwin, and R. J. Molnar, Phys. Rev. B 74, 033302 (2006).

${ }^{22}$ R. Cingolani, A. Botchkarev, H. Tang, H. Morkoc, G. Traetta, G. Coli, M. Lomascolo, A. Di Carlo, F. Della Sala, and P. Lugli, Phys. Rev. B 61, 2711 (2000).

${ }^{23}$ V. I. Litvinov, Phys. Rev. B 68, 155314 (2003).

${ }^{24} \mathrm{M}$. Born and E. Wolf, Principles of Optics (Pergamon, Oxford, 1970).

${ }^{25}$ L. I. Magarill and M. V. Entin, Fiz. Tverd. Tela (Leningrad) 31, 37 (1989) [Sov. Phys. Solid State 31, 1299 (1989)].

${ }^{26}$ E. L. Ivchenko and G. E. Pikus, Superlattices and Other Heterostructures, Symmetry and Optical Phenomena, Springer Series in Solid State Sciences Vol. 110 (Springer-Verlag, Heidelberg, 1995); Superlattices and Other Heterostructures, Symmetry and Optical Phenomena, Springer Series in Solid State Sciences Vol. 110, 2nd ed. (Springer-Verlag, Heidelberg, 1997).

${ }^{27}$ N. A. Sinitsyn, Q. Niu, and A. H. MacDonald, Phys. Rev. B 73, 075318 (2006).

${ }^{28}$ S. A. Tarasenko, Pis'ma Zh. Eksp. Teor. Fiz. 85, 216 (2007) [JETP Lett. 85, 182 (2007)].

${ }^{29}$ S. A. Tarasenko, Phys. Rev. B 73, 115317 (2006).

${ }^{30}$ E. V. Beregulin, S. D. Ganichev, K. Yu. Glukh, Yu. B. LyandaGeller, and I. D. Yaroshetskii, Fiz. Tverd. Tela (Leningrad) 30, 730 (1988) [Sov. Phys. Solid State 30, 418 (1988)].

${ }^{31}$ U. Rössler and J. Kainz, Solid State Commun. 121, 313 (2002).

${ }^{32}$ L. E. Golub and E. L. Ivchenko, Phys. Rev. B 69, 115333 (2004).

${ }^{33}$ E. V. Beregulin, S. D. Ganichev, K. Yu. Gloukh, Yu. B. LyandaGeller, and I. D. Yaroshetskii, Fiz. Tverd. Tela (Leningrad) 31, 115 (1989) [Sov. Phys. Solid State 31, 63 (1989)].

${ }^{34}$ G. L. Bir and G. E. Pikus, Symmetry and Strain-Induced Effects in Semiconductors (Wiley, New York, 1974).

${ }^{35}$ Zhongqin Yang and Zhizhong Xu, Phys. Rev. B 54, 17577 (1996).

${ }^{36}$ R. Beresford, J. Appl. Phys. 95, 6216 (2004). 